\title{
Microbial Profile of Blood Stream Infections and their Antibiotic Susceptibility Pattern of Isolates among Paediatric Patients admitted in a Teaching Hospital of West Bengal
}

\author{
Nishant Kumar, Retina Paul* and Kuhu Pal \\ Department of Microbiology, College of Medicine \& JNM Hospital, WBUHS, \\ Kalyani, West Bengal, India \\ *Corresponding author
}

Keywords

Blood stream infections,

Methicillin resistant

Staphylococcus aureus, Grampositive cocci, Gram-negative bacilli

Article Info

Accepted:

20 January 2020

Available Online:

10 February 2020

\section{A B S T R A C T}

Blood stream infections (BSI) are a global burden in developing countries. Increased incidence leads to morbidity and mortality in neonates as well as Paediatric age group patients. Blood culture is an effective tool for diagnosis of BSI. Aim is to determine various blood stream pathogens and their antibiotic susceptibility pattern. Blood Samples were received during February to August 2018 in JNM hospital of age group up to 12 years and incubated at BacT/ ALERT 3D System. Positives were subcultured on Blood and MacConkey agar and incubated at $37{ }^{\circ} \mathrm{C}$ for $18-24 \mathrm{hrs}$. Standard biochemical test and antibiotic susceptibility test (AST) were performed according to CLSI guidelines. Among 140 Blood samples, $73(52.1 \%)$ were culture positive among the positive samples $36(49.3 \%)$ in neonates, $13(17.8 \%)$ in infants, and $24(32.9 \%)$ in Paediatric patients. 39 (53.4\%) isolates were Gram-positive cocci (GPC) and 25 (35.6\%) were Gram-negative bacilli (GNB) and 8 (11\%) were Candida spp. Among GPC, Staphylococcus aureus33 (45.83\%) were predominant isolates. Out of which 14 (42.42\%) were Methicillin resistant Staphylococcus aureus (MRSA). While among GNB most frequent isolates were Pseudomonas aeruginosa 9 (12.3\%) followed by Acinetobacter spp $6(8.2 \%)$. Multi Drug Resistant (MDR) was observed in GNB. We can conclude from our study that changing trends of microbial isolates and continuous monitoring of antibiotic susceptibility pattern may help clinicians to choose wisely the empirical therapy for responsible pathogen of BSI.

\section{Introduction}

Blood stream Infections (BSI) are one of the major problems in developing countries and its increased incidence leads to morbidity and mortality in neonates as well as children. It happens due to their weak immune status during birth. ${ }^{[1]}$ Detection of microbes done by 
appropriate blood culture and blood should be collected aseptically before starting antibiotics. ${ }^{[2]}$ Bacteraemia, refers to the presence of bacteria in blood which leads to colonization of the blood circulation which are not generally said to be a risky. This is due to numerous physiological conditions give rise to transient bacteraemia without any obvious clinical sequelae. ${ }^{[3]}$ Microorganisms present in blood stream continuously or intermittently are threat to every organ in the body. ${ }^{[4]}$ BSI has become the major problem for mortality among children's specially if caused by Multi drug resistant (MDR) organisms and they are more likely to extend the hospital stay, possibly life threatening, increase the risk of death and require treatment with more costly antibiotics. Both Gram positive and Gramnegative bacteria causes bacteraemia and septicemia. Gram negative septicemia, also known as endotoxic shock, which is more severe than Gram positive septicemia. ${ }^{[5]}$ The growing frequency of antimicrobial resistance among microbial pathogens causing nosocomial and community acquired infections is making numerous classes of antimicrobial agents effective resulting in emergence of antimicrobial resistance. ${ }^{[6]}$ So, Blood culture is very useful for rapid identification and antibiotics susceptibility testing of the causative pathogen that helps the clinicians to start the empirical therapy and antibiotic susceptibility reports will also help them to know about the resistance pattern of the particular drugs and it will help them to choose wisely. Therefore, in this study which aimed to determine the common microbial agents of BSI and to determine antibiotic susceptibility patterns among paediatric patients visiting in Teaching Hospital of West Bengal.

\section{Materials and Methods}

The Present Prospective study was conducted at Microbiology department of a teaching hospital west Bengal, eastern India during
February to August 2018. A total of 140 blood culture bottle samples were received in Microbiology department from S.N.C.U and Paediatrics wards of JNM hospital. The blood samples were first incubated in BacT/ALERT 3D an automated blood culture system which uses colorimetric principle for microbial detection in presence of any microbes BacT/ALERT 3D gives automatically response in machine, with help of sterile syringe the blood bottles were inoculated in Blood and MacConkey agar and it is incubated at $37{ }^{\circ} \mathrm{C}$ in BOD incubator. Then for colony morphology it was studied using Gram stain. Then standard biochemical test were performed. ${ }^{[7]}$ The Antibiotic Susceptibility testing were performed by Kirby-Bauer's disc diffusion method following by CLSI guidelines. For GPC Following AST discs were used Penicillin (10 units), Piperacillin-tazobactam $(100 / 10 \mu \mathrm{g})$, Azithromycin and Erythromycin (15 $\mu \mathrm{g})$, Tetracycline, Amikacin, Cefepime, Cefuroxime, Ceftriaxone, Cefoxitin, Cefotaxime, Chloramphenicol and Linezolid $(30 \mu \mathrm{g})$, Gentamicin and Meropenem $(10 \mu \mathrm{g})$, Ciprofloxacin, Ofloxacin and Levofloxacin (5 $\mu \mathrm{g})$. And for GNB following AST disc were used Piperacillin $(100 \mu \mathrm{g})$, Amoxicillinclavulanate $\quad(20 / 10 \quad \mu g), \quad$ Piperacillintazobactam $(100 / 10 \mu \mathrm{g}), \quad$ Tetracycline, Cefixime, Cefuroxime, Ceftriaxone, Cefotaxime, Cefepime, Cefoxitin, Ceftazidime, Aztreonam, Netilmicin, Chloramphenicol and Amikacin (30 $\mu \mathrm{g})$, Meropenem and Gentamicin (10 $\mu \mathrm{g})$, Ciprofloxacin, Levofloxacin, and Ofloxacin (5 $\mu \mathrm{g}) .{ }^{[8]}$

\section{MRSA test for Staphylococcus aureus}

The MRSA test were done by Cefoxitin Disk diffusion agar method on Muller Hinton agar following the CLSI guidelines the cut-offs of MRSA sensitive and resistant zones size were $\geq 22$ and $\leq 21 \mathrm{~mm}^{[8]}$ 


\section{ESBL test among Enterobacteriaceae}

The ESBL test was done on Muller Hinton agar by Kirby-Bauer's disc diffusion method. The Cut-offs zone size for screening of ESBLs test was done by according to CLSI guidelines. K. pneumoniae, E. coli were, e.g., ceftazidime, $\leq 25 \mathrm{~mm}$. While for $P$. mirabilis ceftazidime, $\leq 22 \mathrm{~mm}$. and confirmatory test was done by placing a disk of ceftazidime $(30$ $\mu \mathrm{g})$ alone and ceftazidime + clavulanic acid $(30 / 10 \mu \mathrm{g})$ or on a Mueller-Hinton Agar plate with minimum distance $20 \mathrm{~mm}$ apart from each other. After overnight incubation plates were examined. $A \geq 5 \mathrm{~mm}$ increase in a zone diameter for either antimicrobial tested in combination with clavulanate vs the zone diameter of the agent when tested alone which indicates the ESBL producers. (For Exampleceftazidime zone $=16$; Ceftazidimeclavulanate zone $=21) .{ }^{[8]}$ (Fig. 1$)$.

\section{D- Test among Staphylococcus aureus}

The D- test were performed on muller Hinton agar by Kirby-Bauer's disc diffusion method by placing the Erythromycin disc at a distance of $15 \mathrm{~mm}$ (edge to edge) from clindamycin disc incubated at $37{ }^{\circ} \mathrm{C}$ overnight. Flattening of (D shaped around clindamycin in the area between two disc indicated inducible clindamycin resistance). ${ }^{[8]}$ (Fig. II)

Data were analysed by SPSS ver. 25 (IBM) using chi square test and $\mathrm{p}$ value $\leq 0.05$ were considered as statistically significant.

\section{Results and Discussion}

Out of 140 blood culture samples processed, $73(52.1 \%)$ were Culture positive while 67 $(47.9 \%)$ were culture negative and Blood culture positivity was $36(49.3 \%)$ in neonates (0-28 days), and $13(17.8 \%)$ in infants (1 months- 1 years), 24 (32.9\%) in Pediatrics (112 years). In this study Male were predominant $45(61.6 \%)$ while Females were $28(38.4 \%)$ isolates and among 45 males the most frequent isolates were occurred among males in both neonatal $23(51.1 \%)$ and Peadiatric $14(31.1 \%)$ age group as well as infants $8(17.7 \%)$ children (Table 1$)$.

Among 73 positive samples 37 (50.7\%) sample isolated were from S.N.C.U Wards. While $36(49.3 \%)$ sample isolated were from Pediatrics wards. From positive samples in direct microscopy 39 (53.4\%) isolates were Gram-positive Cocci (GPC) and GramNegative Bacilli were 26 (35.6\%) while candida was few in number i.e. 08 (11\%). Among the neonates the most frequent isolates belong to Gram Negative Bacilli 22 (57.89\%) while among the paediatrics age group children the most frequent isolates were belongs to the Gram-positive cocci 29 $(82.85 \%)$ (Table 2). Among total 45 positive isolates from Males and 28 isolates from females. The males were most commonly affected which includes both Gram-positive cocci and bacilli infections 24 (53.3\%) and 17 $(37.7 \%)$ and candida $4(8.8 \%)$. While in females Gram positive isolates $15(53.5 \%)$ were much higher than Gram-negative bacilli infections $9(32.14 \%)$ and candida 4 (14.28 $\%)$. But for the GPC infections male 24 (53.3\%) and females 15 (53.5\%) were affected same.

Among Neonates age group from S.N.C.U wards the predominant organisms were Pseudomonas aeruginosa, candida spp., Acinetobacter spp., and Staphylococcus aureus while among paediatrics agegroup from paediatrics wards. most frequent organisms were Staphylococcus aureus. Over all total 73 positive isolates Staphylococcus aureus remain highest isolates 33 (45.2\%) followed by Pseudomonas spp.9 (12.3\%), Candida spp. 8 (11\%), and rest isolates was 1$8 \%$ (Table 3). Among 45 positive isolates from Males and 28 from females the 
Staphylococcus aureus were the most frequent isolates which was $20(44.4 \%)$ and $13(46.4 \%)$ in males and females respectively.

Among 39 total GPC isolates 38 were Staphylococcus Species and 01 were Streptococcus pyogenes while among 38 Staphylococcus Species 33 were Staphylococcus aureus and 05 of them are Coagulase Negative Staphylococcus. Out of which 01 were Staphylococcus epidermidis and other were 02 Staphylococcus saprophyticus which has not been isolated on repeat sample so they are considered as nonpathogenic. and rest 02 isolates were Micrococcus spp. whose antibiotics susceptibility pattern has not given because they are considered as a normal commensal flora of skin. The most frequent bacterium Staphylococcus aureus $(\mathrm{n}=33)$ were showed higher sensitivity towards linezolid $100 \%$ followed by Tetracycline $90.3 \%$, Chloramphenicol 87.1\%, Clindamycin $84.8 \%$ Gentamycin $74.1 \%$. While sensitivity of Levofloxacin $58.1 \%$, Ofloxacin $57.4 \%$ and $\beta$ lactam antimicrobial agents including Cephalosporin, Carbapenem responded moderately $57.4 \%$ (Fig. III).

Among 33 isolated Staphylococcus aureus $57.5 \%(\mathrm{n}=19)$ were the MSSA (Methicillin Sensitive Staphylococcus aureus), while 42.5 $\% \quad(n=14)$ were the MRSA (Methicillin Resistant Staphylococcus aureus). Out of 33 samples of S. aureus, 04 (12.12\%) samples showed D-test positive (inducible clindamycin resistance) and 14 (42.42\%) showed D-test negative (MS phenotype). Constitutive MLSB phenotypes were 6 (18.18\%) (Table 4).

Among Enterobacteriaceae $(n=11)$ the highest resistance shows among antibiotics Tetracycline, Cefepime $81.8 \%$, followed by cefotaxime, ceftazidime, Amikacin $72.7 \%$,
Aztreonam and cefoxitin 63.6\%. While cefixime, Netilmicin, Gentamicin were $54.5 \%$, Ciprofloxacin, meropenem and Piperacillin tazobactam $45.5 \%$, Amoxicillin clavulanate $36.4 \%$, Chloramphenicol shows least resistance $18.2 \%$ (Fig. IV).

Out of 11 Enterobacteriaceae 7 isolates (3 Proteus Mirabilis, 3 Klebsiella pneumoniae, and $1 \mathrm{E}$. coli) were tested for ESBLs and 3 $(42.85 \%)$ isolates which was 1 E. coli and 1 Klebsiella. pneumoniae and 1 Proteus. mirabilis belongs to the ESBL producers.

Among 11 candida isolates which were isolated on direct microscopy and colony morphology their antibiotic susceptibility pattern hasn't given as they are considered as normal skin commensals. Which has been treated as commensals on a repeat isolation. Among Pseudomonas aeruginosa $(\mathrm{n}=9)$ Highest susceptible towards the PiperacillinTazobactam, Aztreonam 66.7\%, Amikacin 55.6\%. While Piperacillin, Ceftazidime, ciprofloxacin $44.4 \%$, Gentamicin, cefepime Levofloxacin, Netilmicin and ofloxacin shows least susceptible $33.3 \%$ (Fig. V).

Among Acinetobacter spp $(n=6)$, highest resistance shows among Amikacin, Gentamicin, Netilmicin, and levofloxacin, Ciprofloxacin, meropenem 100\%. Followed by cefixime and ceftriaxone $50 \%$ while cefepime and cefotaxime $66.7 \%$ while least resistance was occurred in piperacillintazobactam $33.3 \%$ (Fig. VI). Multidrug resistance was observed among Acinetobacter spp. (Fig. VII).

In developing countries like India BSI remains a major cause of morbidity and mortality despite of having major role in terms of advances in diagnosis as well as treatment facility in health care setup. 
Table.1 Gender wise distribution of positive blood culture age groups children

\begin{tabular}{|c|c|c|c|}
\hline Group & Male & Female & Total \\
\hline Neonates & 23 & 13 & 36 \\
\hline Infants & 8 & 5 & 13 \\
\hline Pediatrics & 14 & 10 & 24 \\
\hline Total $\left(\mathbf{p}=\mathbf{0 . 9 1}, \boldsymbol{\chi}^{\mathbf{2}}=\mathbf{0 . 1 8}\right)$ & $\mathbf{4 5}$ & $\mathbf{2 8}$ & $\mathbf{7 3}$ \\
\hline
\end{tabular}

Table.2 Distribution of age group children with Microscopic findings

\begin{tabular}{|c|c|c|c|c|}
\hline Ward & GPC & GNB & Candida & Total \\
\hline Neonatal & 10 & 22 & 6 & 38 \\
\hline Paediatrics & 29 & 4 & 2 & 35 \\
\hline Total $\left(\mathbf{p}<\mathbf{0 . 0 0 1}, \boldsymbol{\chi}^{\mathbf{2}=\mathbf{2 3 . 6 3 5}}\right)$ & $\mathbf{3 9}$ & $\mathbf{2 6}$ & $\mathbf{8}$ & $\mathbf{7 3}$ \\
\hline
\end{tabular}

Table.3 Distribution of isolates with age group of children

\begin{tabular}{|c|c|c|c|c|}
\hline Isolates & Neonates & Infants & Pediatrics & Total \\
\hline S. aureus & 5 & 10 & 18 & 33 \\
\hline E. coli & 1 & 0 & 0 & 1 \\
\hline Klebsiella pneumoniae & 3 & 0 & 0 & 3 \\
\hline Proteus mirabilis & 3 & 0 & 0 & 3 \\
\hline Acinetobacter spp. & 6 & 0 & 0 & 6 \\
\hline Pseudomonas aeruginosa & 6 & 1 & 2 & 9 \\
\hline Streptococcus pyogenes & 1 & 0 & 0 & 1 \\
\hline Candida spp. & 6 & 0 & 2 & 8 \\
\hline Citrobacter Spp. & 3 & 0 & 1 & 4 \\
\hline CoNS & 2 & 2 & 1 & 5 \\
\hline Total $\left(\mathbf{p}=\mathbf{0 . 0 0 6}, \chi^{\mathbf{2}}=\mathbf{3 6 . 5 8 4}\right)$ & $\mathbf{3 6}$ & $\mathbf{1 3}$ & $\mathbf{2 4}$ & $\mathbf{7 3}$ \\
\hline
\end{tabular}

Table.4 Phenotypic D test among Staphylococcus aureus

\begin{tabular}{|c|c|c|}
\hline SUSCEPTIBILITY PATTERN (PHENOTYPE) & NO. OF ISOLATES & $\begin{array}{c}\text { PERCENTAGE } \\
(\boldsymbol{\%})\end{array}$ \\
\hline $\begin{array}{c}\text { ER-R and CD-S (D- } \\
\text { TEST POSITIVE iMLSB) }\end{array}$ & 04 & 12.12 \\
\hline $\begin{array}{c}\text { ER-R and CD-S (D-TEST } \\
\text { NEGATIVE, MS) }\end{array}$ & 14 & 42.42 \\
$\begin{array}{c}\text { ER-R and CD-R } \\
\text { (CONSTITUTIVE } \\
\text { MLSB) }\end{array}$ & 6 & 18.18 \\
\hline ER-S and CD-S & & \\
\hline TOTAL & 9 & 27.28 \\
\hline
\end{tabular}


Fig.1 ESBL test in Enterobacteriaceae

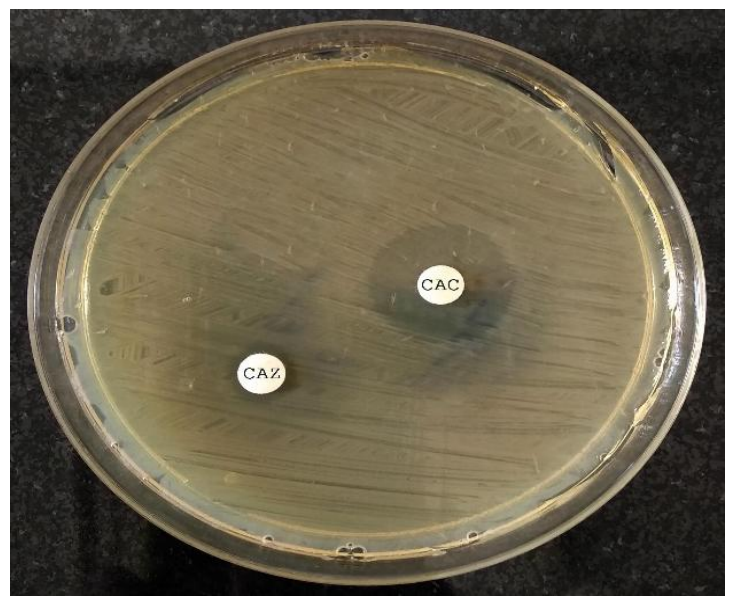

Fig.2 D-test in Staphylococcus aureus

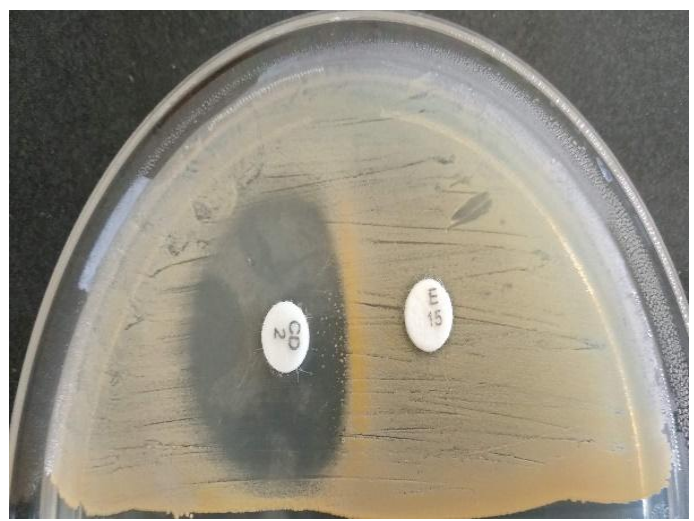

Fig.3 Bar graph showing Antibiotic Susceptibility pattern in Staphylococcus aureus in \%

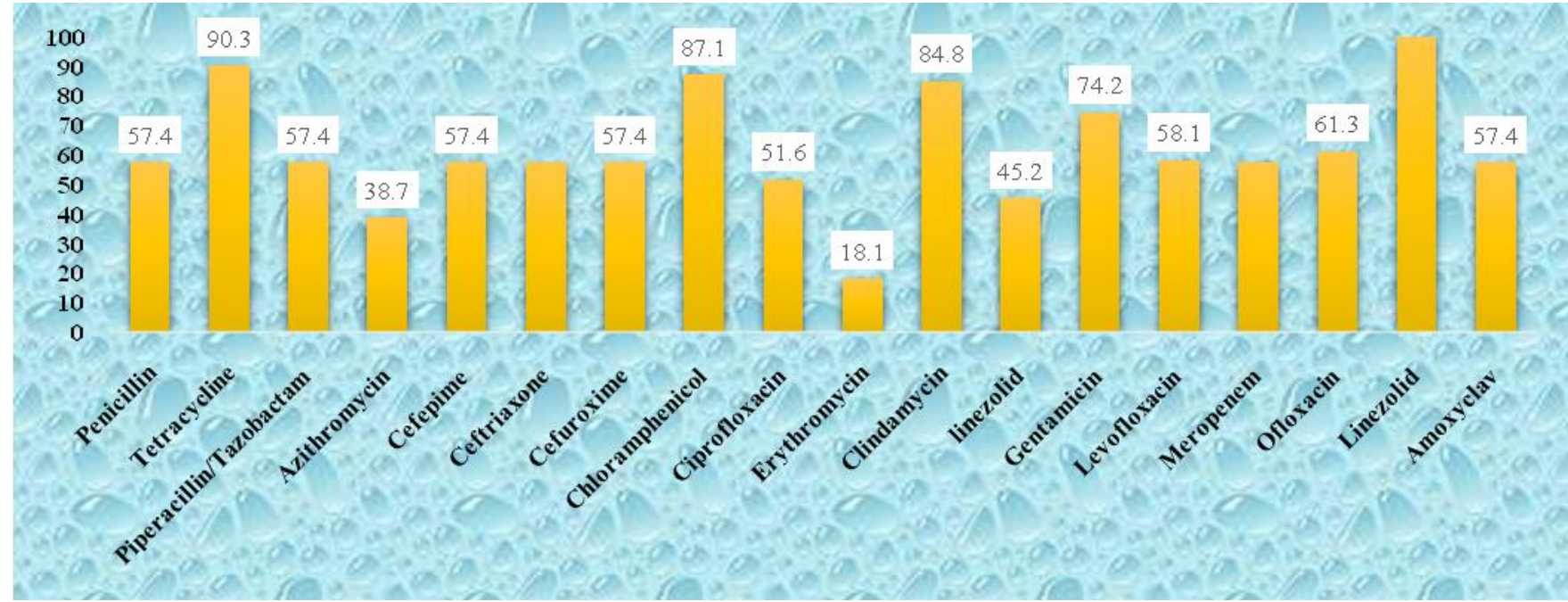


Fig.4 Bar graph showing antibiotic resistance pattern in Enterobacteriaceae in \%

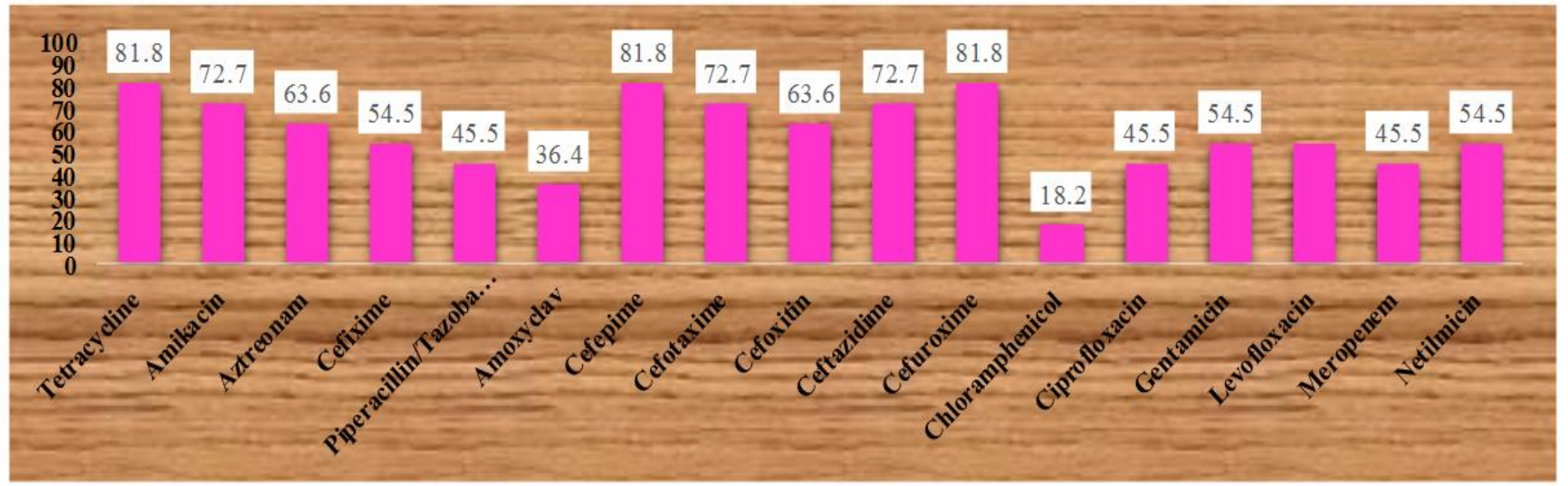

Fig.5 Bar graph showing antibiotic susceptibility pattern among Pseudomonas aeruginosa in \%

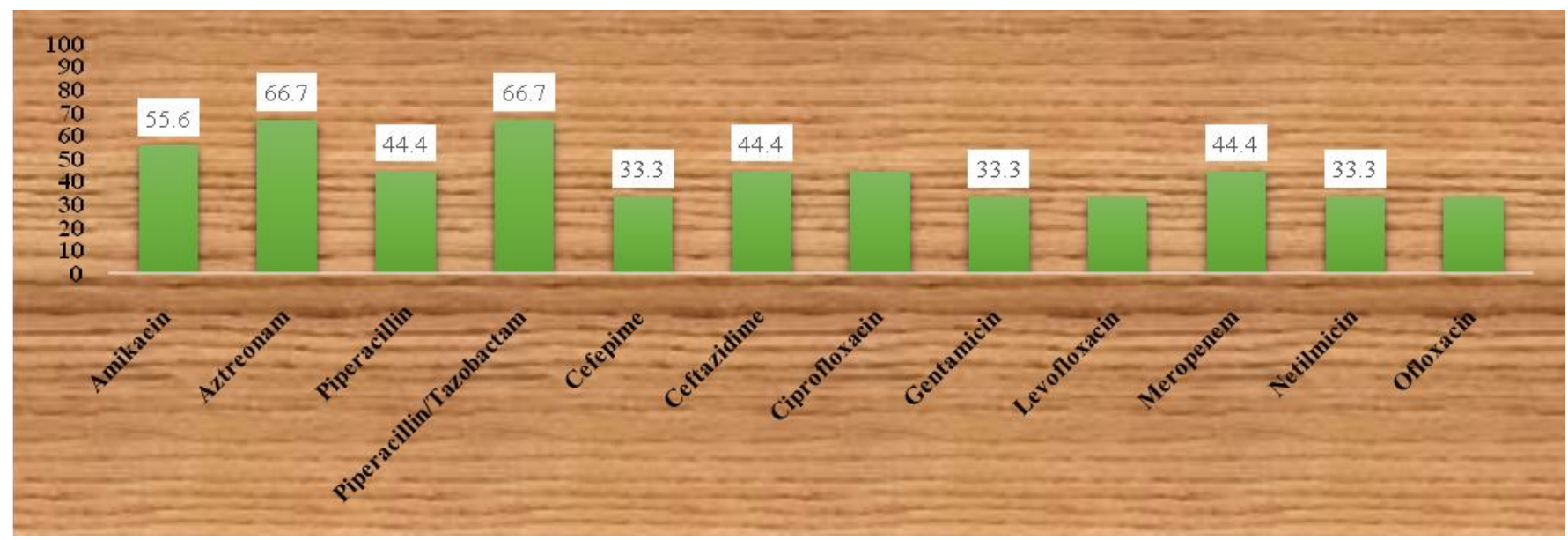

Fig.6 Bar graph showing antibiotic resistance pattern among Acinetobacter spp. in \%

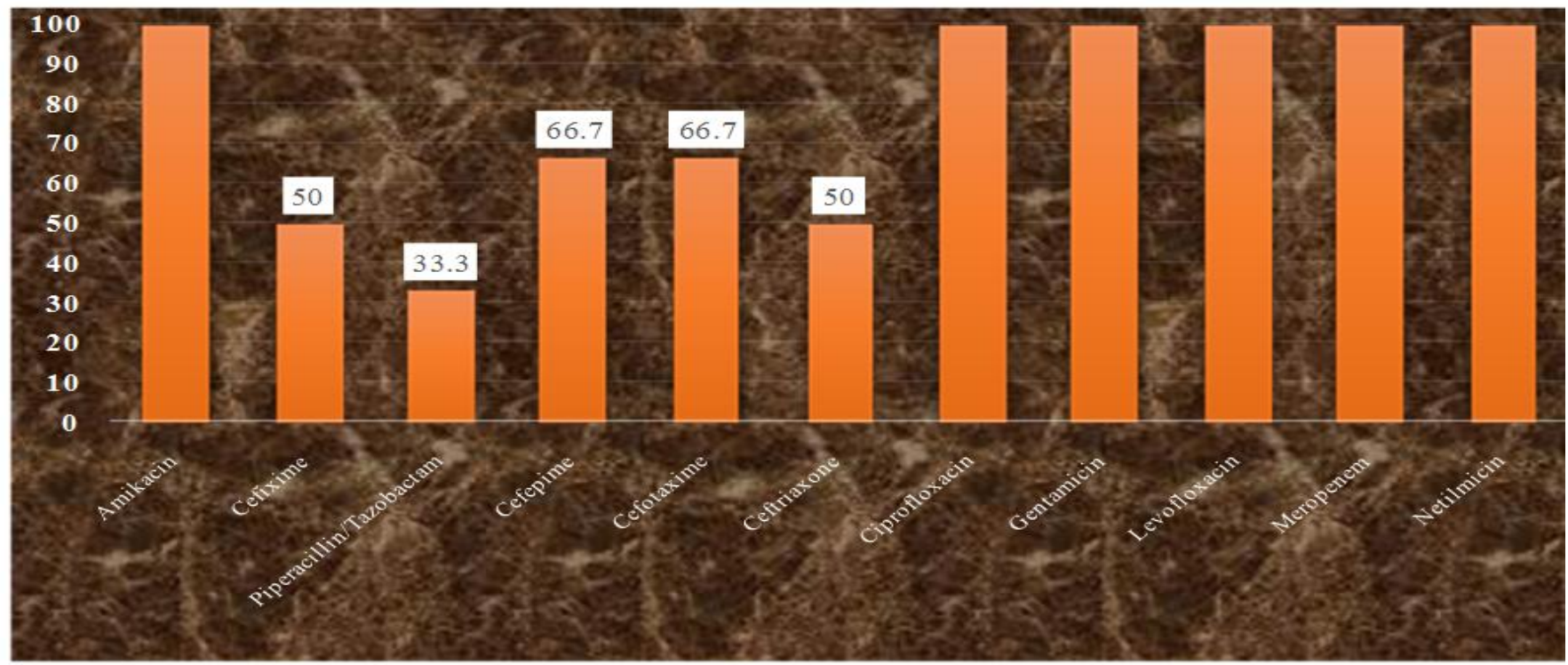


Fig.7 MDR in Acinetobacter spp.

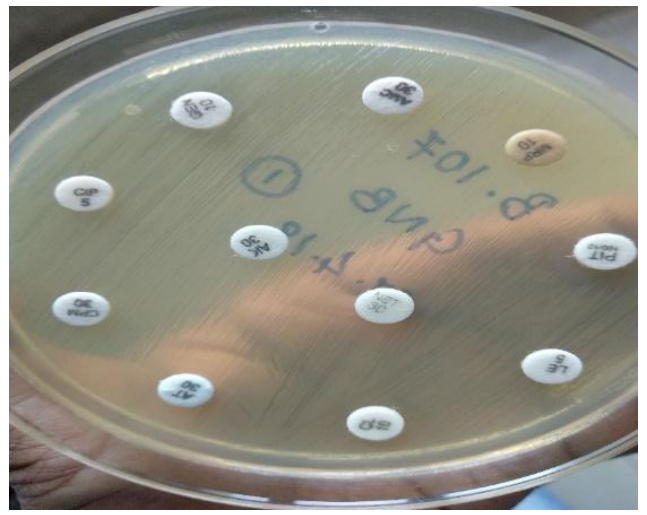

For detection of infectious disease blood culture is considered as a well diagnostic procedure in microbiology. In India there are a lot of misuse of antibiotics by means of selling over the counter in medical hall, or by using the early broad-spectrum antibiotics without having laboratory confirmation of particular infectious disease. So, blood culture has great role and providing the Antimicrobial susceptibility pattern will help the clinicians to start empirical therapy as well as help them to get idea about resistance pattern of particular drugs.

In the present study 140 blood samples were tested to identify the presence of microorganisms in the blood samples. A culture positivity rate in this study $52 \%$ which was lesser than Pakistan 68.4\% (Alaa), and were slightly higher than an Indian study is 40\% (Neuma and Chitnis), Gambia $34 \%$ (Philip et al., ), Zimbabwe 37.1\% (Obi and Mazarua), Nepal 23.1\% (Amatya et al.,) Ethopia 24.2 \% (Ali and Kebede) India 20.5\% (Atul Garg et al., $)^{[9-14,5]}$. This variation might be due to the strains of particular geographical reasons or type of blood culture used or may be due to the antibiotics policy in particular health care setup.

Among 73 positive isolates male were predominant in this study which is $61.6 \%$ in males while $38.4 \%$ in female and similar results also achieved by Mahesh C. $62 \%$ and $38 \%$, Begum et al., $65 \%$ and 27\% Sheresth et al., $63.7 \%$ and $36.3 \%$, Chitralekha et al., $65.6 \%$, and $34.4 \%$ Mohammad A $55.8 \%$ and $44.2 \%$ of males and females respectively. ${ }^{[15-}$ ${ }^{19]}$ There is no exact reason for the male preponderance but from the above studies males were affected most but this could be due to sex-dependent factors ${ }^{[15]}$. The synthesis of gamma globulins is probably regulated by $\mathrm{X}$ - linked immunoregulatory genes and as males are having one $\mathrm{X}$ chromosome, that's why they are more prone for neonatal septicemia than females. ${ }^{[20]}$

In this study the rate of isolation was found to be highest among newborn admitted in SNCU ward i.e. $52 \%$ and in paediatric age group $48 \%$.The frequency of isolation of Gram positive and Gram-negative bacteria in this study were $53.4 \%$ and $35.6 \%$ respectively. Which can be compared for GPC and GNB with studies by Chitralekha S et al., $43 \%$ and $57 \%$ and by Negussie et al., $46.4 \%$ and 51.8 $\%$ and Qureshi et al., $40 \%$ and $60 \%$ respectively. ${ }^{[18,1,3]}$. In this study total 26 GNB were isolated from 38 neonatal wards and among them 22 GNB infections were predominant in new-born. As neonates are more susceptible to develop GNB infections. In this study a significant association were found among the positive age group children and with microscopic findings $(\mathrm{p}<0.001)$. 
The high rates among neonates may be related to immunity or may be the practice of medical device or due to geographical variations.

The most frequent GPC bacteria isolated in this study were Staphylococcus aureus $45 \%$ which was much lesser than study by Mohammad A (86\%). In this study a significant association were found among the positive age group children and with isolates $(p=0.006)$. The increased rates of isolation of Staphylococcal bacteraemia may be related with use of intravascular catheters. ${ }^{[19]}$

Based on susceptibility test in the present study. The sensitivity among Staphylococcus aureus were sensitive to Tetracycline $90.3 \%$ Gentamicin 74.2\%, Ciprofloxacin 51.6\%, Penicillin 57.4\%, Ceftriaxone $57.4 \%$, which can compare studies by Negussie et al., Penicillin 92.3\%, Tetracycline $53.8 \%$ and Qureshi et al., Ciprofloxacin 80\%, Gentamicin 66.6\%. And Mohmmad A et al., Gentamicin 60.6\%, Ceftriaxone 44.9\%, Ciprofloxacin 79.25\%. [1,3,19] while Cephalosporins $\beta$ lactam antimicrobial agents were responded moderately $57.4 \%$ these were due to the MRSA $42.5 \%$ infections Staphylococcus aureus in comparison of MRSA and MRSA study concluded by Kaede $\mathrm{V}$ et al., our results are similar with them 43 $\%$ and $57 \%$. ${ }^{[21]}$ This may be due to the practice of cloxacillin or others antibiotic usage policy. In our study majority of the isolates of Staphylococcus aureus were resistant to erythromycin $81.82 \%$ and sensitive to clindamycin $84.85 \%$ which was higher than Prabhu et al., 28.42\% and 58.6\%. In this study $12.12 \%$ were inducible clindamycin resistant phenotype which was similar results by them i.e., $10.52 \%$. The percentage of MS phenotype and constitutive phenotype of this study is also differing from them. In this study MS phenotype were $24.42 \%$ and $18.18 \%$ were constitutive phenotype which was higher than their study i.e., $8.42 \%$ and $9.47 \%$ respectively. ${ }^{[22]}$

While among Enterobacteriaceae Resistance to Tetracycline $81.8 \%$, Ciprofloxacin and Gentamicin 54.5\%, Chloramphenicol 18.2\%, Ceftazidime and Amikacin 72.7\%, Aztreonam $63.6 \%$, and Tetracycline $81.8 \%$ which can be concluded study by Negussie et al., $8.3 \%$, Ciprofloxacin 0\%, Chloramphenicol 0\%. And Qureshi et al., Ciprofloxacin 50\%, Gentamicin $50 \%$. And by Atul Garg et al., Gentamicin 44.8\%, Amikacin 29.6\%, Ciprofloxacin $42.5 \%$, and Tetracycline 82.4 $\%$.And by Mohammad A et al., Amikacin 61.3\%, Aztreonam 83.4\%, Ceftazidime $58.7 \%$, Ciprofloxacin $8.7 \%$ ). ${ }^{[1,3,5,19,]}$ In this study 7 out of 3 isolates were ESBL producers $43 \%$ which was lesser than Sangare et al., 61.8\%. While in this study Klebsiella pneumoniae and Proteus mirabilis ESBL producers were $66.6 \%$ whileE. coli were $100 \%$ ESBL producers, while they found $41.7 \% .^{[23]}$

In this study Pseudomonas aeruginosa were susceptible to Amikacin $55.6 \%$, Aztreonam and Piperacillin- tazobactam66.7\% and Piperacillin, Ceftazidime and Ciprofloxacin $44.4 \%$, Gentamicin $33.3 \%$ which can be compared by Mohammad A et al., Amikacin 100\%, Aztreonam 33.3\%, Ceftazidime 33.3\%, Ciprofloxacin $100 \%$, Gentamicin $66.6 \%$ and Piperacillin 66.6\%. ${ }^{[19]}$ Among Acinetobacter spp. resistant to Ciprofloxacin and Netilmicin $100 \%$ and cefixime $50 \%$ which can be concluded by Atul Garg et al., $79.4 \%$. And by Mohammad et al., Ciprofloxacin 0\%, Netilmicin $43.4 \%$. ${ }^{[5,19]}$

The limitations in this study as this study were the part of the routine diagnostic test so the Acinetobacter Spp. were remained unidentified for its Speciation due to the lack of VITEK 2 cards during the study period. For the MDR isolates there is need for further screening for ESBL, AmpC, MBLs and 
Carbapenamase resistance test with larger sample size.

We can conclude from this study that a constant monitoring of blood cultures from neonates to Pediatric age group is essential to know that's why Periodical evaluation of the results will enable precautions including complying with antisepsis rules during obtaining samples and also highlights the changing trends of microorganisms and their antibiotic sensitivity pattern. Treatment and administration with antibiotic sensitivity reports instead of antibiotic empirical therapy will reduce the mortality rates and will help the antibiotic usage policy in a health care setup.

\section{Acknowledgements}

The authors would like to acknowledge the technical support by the members of the department of Microbiology, SNCU and Pediatrics units of JNM Hospital Kalyani.

\section{References}

1. Negussie A et al., 2015. Bacteriological profile and antimicrobial susceptibility pattern of blood culture isolates among septicemia suspected children in selected hospitals at Addis Ababa. Ethiopia in International journal of biomedical research November. 6(1): 4709-17.

2. William JH and Max S. 1998, Bacteraemia, septicemia and endocarditis. In: William JH, Max Sussman. Topley and Wilsons Microbiology and Microbial Infections, Vol 3. 11th eds., London; pp. 178-7.

3. Qureshi M and Aziz F. 2011 Prevalence of microbial isolates in blood cultures and their antimicrobial susceptibility profiles. Biomedicajune-Dec. 27(6): 1369.

4. Usha Arora, Pushpa Devi 2007Bacterial
Profile of Blood Stream Infections and Antibiotic Resistance Pattern of Isolates JK SCIENCE October-December; 9(4):186-190.

5. A Garg, S Anupurba, and J Garg.2007 "Bacteriological profile and antimicrobial resistance of blood culture isolates from a university hospital," Journal of Indian Academy of Clinical Medicine. 8(2):139-143.

6. Simkhada P, Raj S K. C., Lamichhane S, Subedi $S$ and Thapa Shrestha U. 2016Bacteriological Profile and Antibiotic Susceptibility Pattern of Blood Culture Isolates from Patients Visiting Tertiary Care Hospital in Kathmandu, Global Journal of Medical Research: C Microbiology and Pathology. 16(1) ISSN: 2249-4618 and Print ISSN: 0975-5888.

7. Collee JG. Mackie and McCarteny: 2006 Practical Medical Microbiology $14^{\text {th }}$ edition UK: Churchill Livingstone Elsevier.

8. Clinical and laboratory standards institute. 2019Performance standards for antimicrobial susceptibility testing; Seventeenth informational supplement. Vol. M-100 $29^{\text {th }}$ ed. Clinical Laboratory Standards Institute.

9. Alaa H. Al-Charrakh, Ali M. AlMuhana, Zainab H. Al-Saadi,2005 Bacterial Profile of Blood Stream Infections In Children Less Than Three Years Old, J. Babylon Univ., 10(3), March, 481-485.

10. Neuma S, Chitnis DS.1996 Antibiogram study over bacterial isolated from cases of bacteremia. Indian J Med Sci. 50:325329.

11. Philip $\mathrm{CH}$, Charles OO, Usman NAI, Ousman S, Samuel A, Naomi S, et al., 2007Bacteraemia in patients admitted to an urban hospital in West Africa. BMC Infect Dis. 7(2):1-8.

12. Obi CL, Mazarura E. 1996Aerobic 
bacteria isolated from blood cultures of patients and their antibiotic susceptibilities in Harare, Zimbabwe. Cent Afr J Med. 42(12):332- 336.

13. Amatya NM, Sheresha B, Lekhak B. 2007Etiological agents of bacteremia and antibiotic susceptibility pattern in Kathmandu hospital. J Nepal Med Assoc. 46(167):112- 118.

14. Ali J, Kebede Y. 2008Frequency of isolation and antimicrobial susceptibility pattern of bacterial isolation from blood culture in Gondar university hospital. Ethio Med J. 46(2):155-161.

15. Mahesh c, Mary A Alex, Navaneeth BV. 2017Bacteriological profile and antibiotic susceptibility test of blood culture isolates in peadtric patients at tertiary care teaching hospital. International Journal of Medical Microbiology and tropical Disease. JanMarch 3(1):31-6.

16. Begum S, Baki MA, Kundu GK, Islam I, Kumar M, Haque A.2012 Bacteriological profile of neonatal sepsis in a tertiary hospital in Bangladesh. J Bangladesh Coll Phys Surg; 30: 66-70.

17. Shrestha NJ, Subedi KU, Rai GK. 2011Bacteriological profile of neonatal sepsis: A hospital-based study. J Nepal Paediatr Soc. 31(1):1-5.

18. Chitralekhasaikumar Bindu D, Kiran Madhusudhan Praveena R Illamani V 2015Aerobic Microbial Profile and
Antibiotic Susceptibility of Blood Isolates in a Tertiary Care Center. Int. J. Pharm. Sci. Rev. Res. 34(2), September - October; 23, Pages: 135-137.

19. Mohammad A et al., 2010 Bacteremia among Jordanian children at Princess Rahmah Hospital: Pathogens and antimicrobial susceptibility patterns Iranian Journal of Microbiology march; 2(1): 22-6.

20. Mustafa M, Ahmed SL. 2014. Bacteriological profile and antibiotic susceptibility patterns in neonatal septicemia in view of emerging drug resistance. J Med Allied Sci. 4(1):02-08.

21. Kaede V. Sullivan, Nicole, N. turner, Sylvester, S. Roundtree, Karin L. McGowan. 2013 Rapid detection of Methicillin- Resistant Staphylococcus aureus (MRSA) and Methicillinsusceptible Staphylococcus aureus (MSSA) using the key path MRSA/MSSA blood culture test and the BacT/ALERT system in a paediatric population. Arch Pathol lab Med. August; 137:1103-05.

22. Kavitha Prabhu, Sunil Rao, and Venkatakrishna Rao 2011. J Lab Physicians. Jan-Jun; 3(1): 25-27.doi: PMCID: PMC3118052 PMID: 2170165.

23. Sangare et al., 2016. Prevalence of ESBL-producing Enterobacteriaceae isolated from blood cultures in Mali $\mathbf{J}$ Infect Dev Ctries. 10(10):1059-106.

\section{How to cite this article:}

Nishant Kumar, Retina Paul and Kuhu Pal. 2020. Microbial Profile of Blood Stream Infections and their Antibiotic Susceptibility Pattern of Isolates among Paediatric Patients admitted in a Teaching Hospital of West Bengal. Int.J.Curr.Microbiol.App.Sci. 9(02): 2895-2905. doi: https://doi.org/10.20546/ijcmas.2020.902.329 